\title{
Correction Note
}

\section{Correction note:}

This is to notify correction of author name in manuscript: Taalaibek

Kudaiberdiev, Irina Akhmedova, Bakyt Imanov, Damirbek Abibillaev, Zhunus

Kadyraliev, Nazgul Shoonaeva, Chinara Satarova, Gulzada Imanalieva,

Kayirnisa Tilemanbetova, Zarema Zhakypova, Gulnaz Tursunbekova. The results of a three-year research program on assessment of primary

diagnostic issue of congenital heart defects in children under 1 year and the availability of cardiac surgical care in the Kyrgyz Republic.

Heart, Vessels and Transplantation 2019; 3: 74-82 doi:

10.24969/hvt.2019.122 Nazgul Shoonaeva must read Nurgul Shoonaeva.

Editors apologize for this typo mistake. 